Advances in Gene Technology: The Genome and Beyond -

Structural Biology for Medicine (Proceedings of the 2002 Miami

Nature Biotechnology Winter Symposium)

TheScientificWorld 2002, 2(S2), 115-116

ISSN 1532-2246; DOI 10.1100/tsw.2002.54

\title{
PRECURSOR STRUCTURE OF CEPHALOSPORIN ACYLASE: INSIGHTS INTO AUTO- PROTEOLYTIC ACTIVATION IN A NEW N-TERMINAL HYDROLASE FAMILY
}

\author{
Youngsoo Kim ${ }^{\ddagger},,^{*}$, Sanggu Kim ${ }^{\ddagger}$, Thomas N. Earnest ${ }^{\perp}$, and Wim G.J. Hol ${ }^{\S, \|}$ \\ *School of Chemical Engineering, Yeungnam University, Dae-Dong, Kyungsan 712-749, Korea; \\ ${ }^{\perp}$ Berkeley Center for Structural Biology, Physical Biosciences Division, MS6-2100, Lawrence \\ Berkeley National Laboratory, Berkeley, CA 94720; ${ }^{\S}$ Department of Biochemistry, Biomolecular \\ Structure Center, and "Howard Hughes Medical Institute, University of Washington, P. O. Box, \\ 357742, Seattle, WA 98195-7742 \\ *ykim1@yu.ac.kr
}

INTRODUCTION. Autocatalytic proteolytic cleavage is a frequently observed post-translational modification in proteins. Cephalosporin acylase (CA) is a recently identified member of the $\mathrm{N}$ terminal hydrolase family that is activated from an inactive precursor by autoproteolytic processing, generating a new N-terminal residue, which is either a Ser or a $\mathrm{Thr}[1]$. The N-terminal Ser or Thr becomes a nucleophilic catalytic center for intramolecular and intermolecular amide cleavages. The gene structure of the open reading frame of CAs generally consist of a signal peptide followed by the $\alpha$-subunit, a spacer sequence, and the $\beta$-subunit, which are all translated into a single polypeptide chain, the CA precursor. The precursor is post-translationally modified into an active heterodimeric enzyme with $\alpha$ - and $\beta$-subunits, first by intramolecular cleavage and second, by intermolecular cleavage.

METHOD. The structure of the S1 $\beta A$ precursor CA was determined to $2.5 \AA$ resolution (Fig. 1). In the course of the structure refinement, the Fo-Fc difference Fourier map, with all the spacer residues omitted for phase calculation, showed clear positive density for spacer sequence prior to adding any information of these backbone residues in the unbiased map. The 11 residues of the spacer from 159s to 169 s (EGDPPDLADQG) were easily built into the Fo-Fc difference map using $\mathrm{O}$ and were refined using CNS to an Rcryst of $20.3 \%$ and an Rfree of $23.7 \%$.

RESULTS AND DISCUSSION. We solved the first CA precursor structure (Fig. 1) from a class I CA from Pseudomonas diminuta (CAD) at $2.5 \AA$ resolution[2]. A conserved water molecule, stabilized by four hydrogen bonds in unusual pseudo-tetrahedral geometry, plays a key role to assist the OG atom of Ser1 $\beta$ to generate a strong nucleophile (Fig. 2a), which could provide insight into the mechanism of intramolecular cleavage. Thus, we would propose a plausible mechanism for the intramolecular cleavage of precursor CA. Initially, the hydroxyl of Ser1 $\beta$ is assisted by the conserved water (WAT1 in Fig. 2a), which is stabilized by four hydrogen bonds in pseudotetrahedral geometry and may accept a proton from the hydroxyl group. As a result, the hydroxyl of Ser1 $\beta$ is precisely positioned for nucleophilic attack[3]. Eventually, this nucleophilic attack will 
result in a free N-terminal Ser1 $\beta$ and the carboxylate of Gly169s (Fig. 2b).

\section{REFERENCES}

1. $\quad$ Murzin, A.G. (1996) Curr. Opin. Struct. Biol. 6(3), 386-394.

2. Kim, Y., Yoon, K.H., Khang, Y., Turley, S., and Hol, W.J.G. (2000) Structure 8, 1059-1068.

3. Xu, Q., Buckley, D., Guan, C., and Guo, H.-C. (1999) Cell 98, 651-661. 


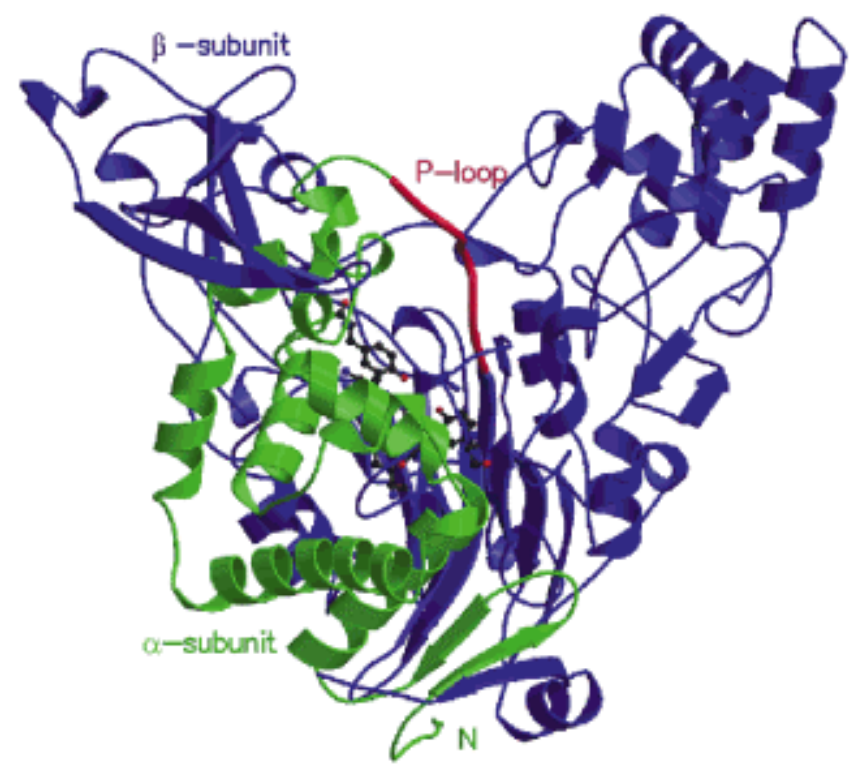

FIGURE 1. Structure of precursor CA. The precursor structure is a single chain protein, consisting of the $\alpha$ subunit of active CAD in green, an orange colored P-loop (spacer structure in loop form), and the $\beta$-subunit of CAD in purple. The side-chain pocket for binding the substrate glutaryl-7aminocephalosporanic acid is represented by a ball-and stick model in the center[2].

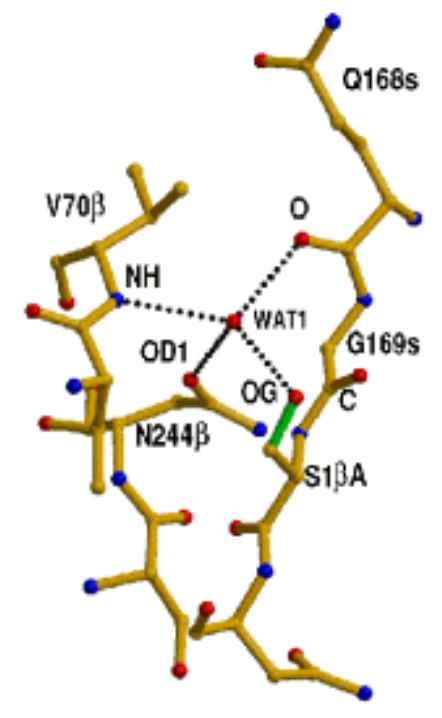

a 


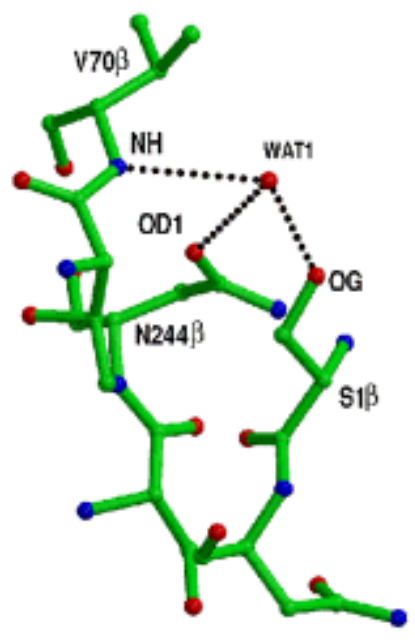

b

FIGURE 2. Hydrogen bonding of a conserved water in precursor CA and active CA. The dotted lines represents hydrogen bonds ranging in 2.8-2.9 $\AA$. (a) The conserved water of precursor CA. The OG atom of Ser $1 \beta$ in the S1 $\beta A$ precursor CA was modeled after the torsional geometry of the OG atom of Ser $1 \beta$ residue of CA structure[2]. (b) The conserved water of CA. The conserved WAT1 is hydrogen bonded to 2 proton acceptors and 1 donor, which are also involved in chemical catalysis of $\mathrm{CA}[2]$. 

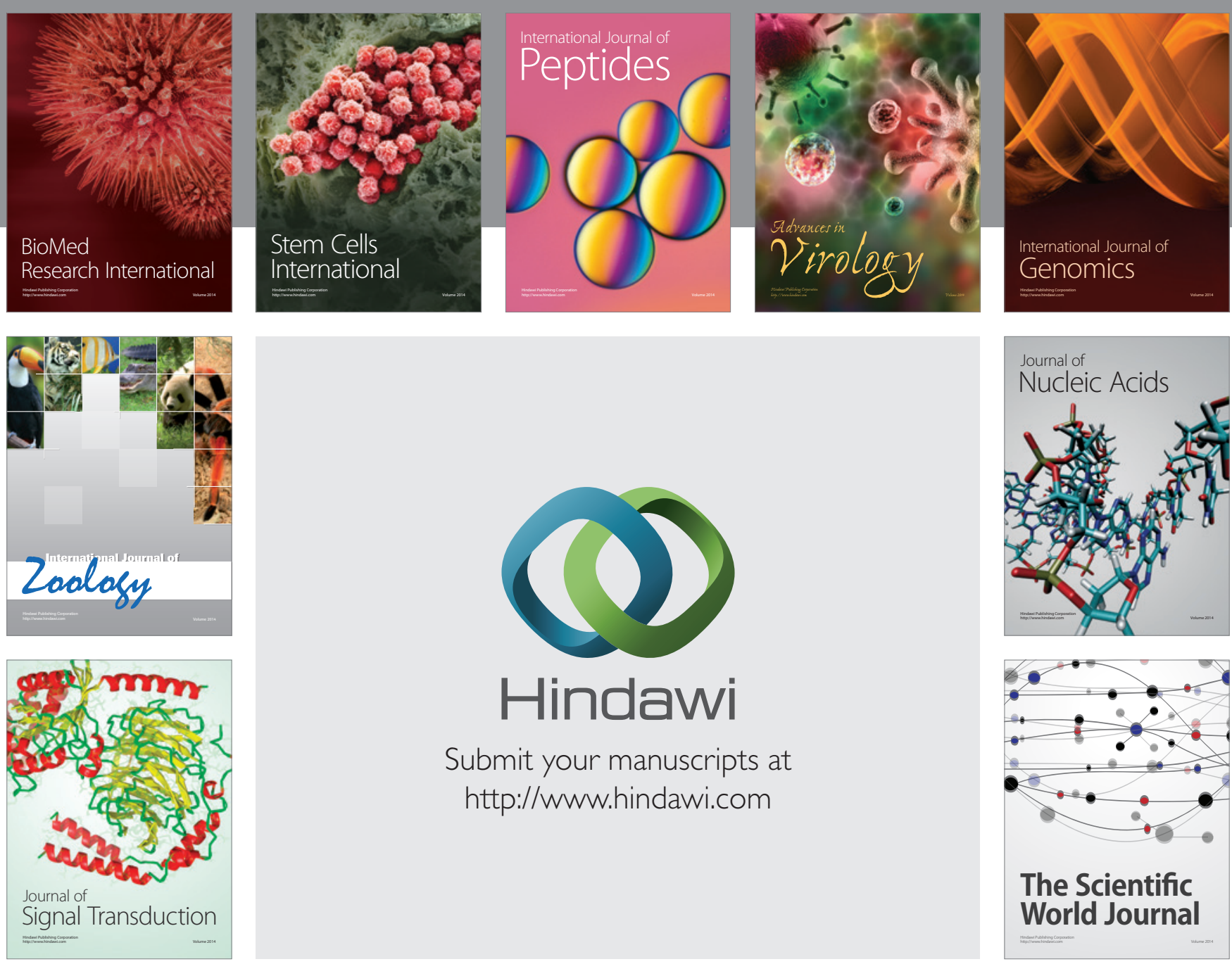

Submit your manuscripts at

http://www.hindawi.com
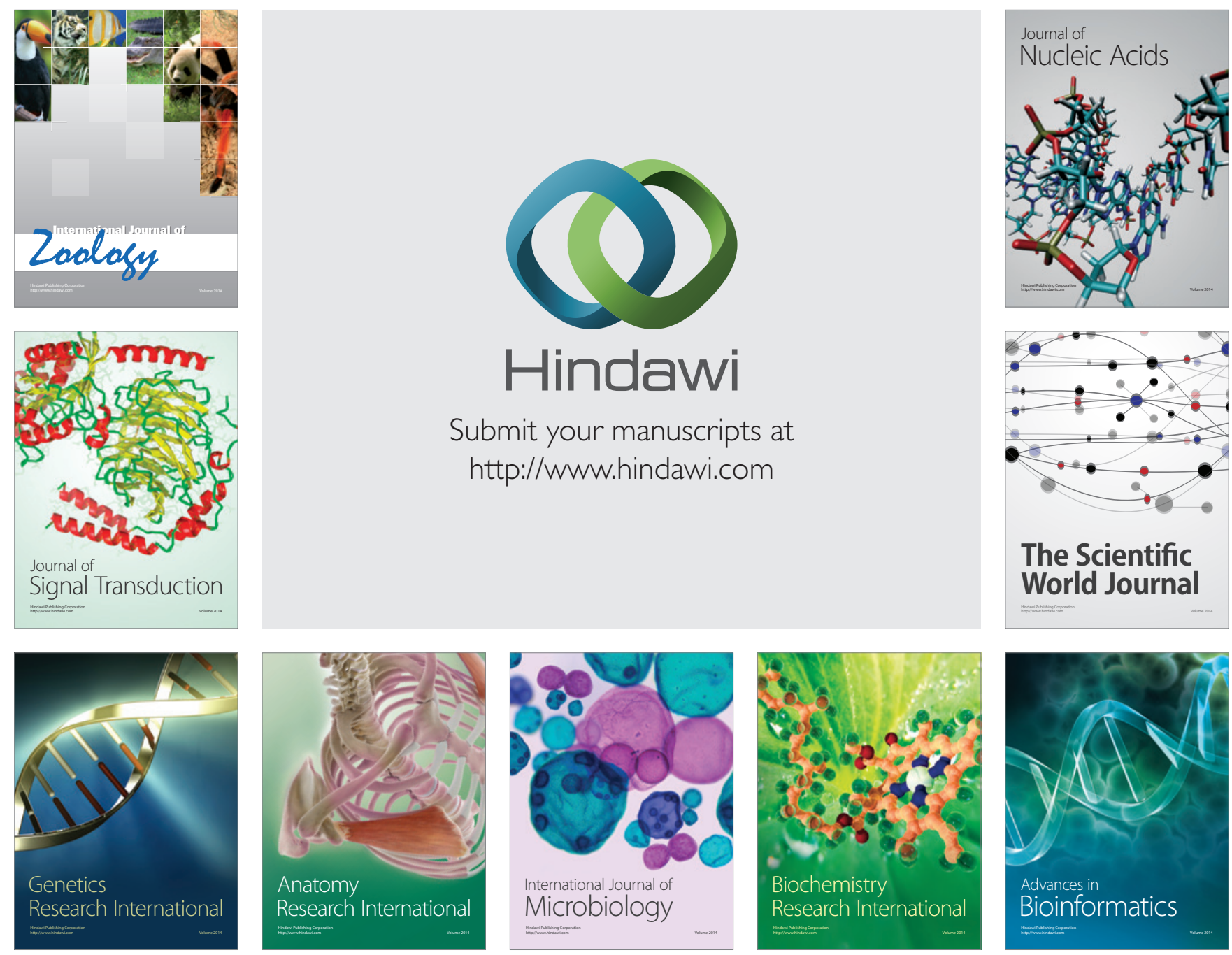

The Scientific World Journal
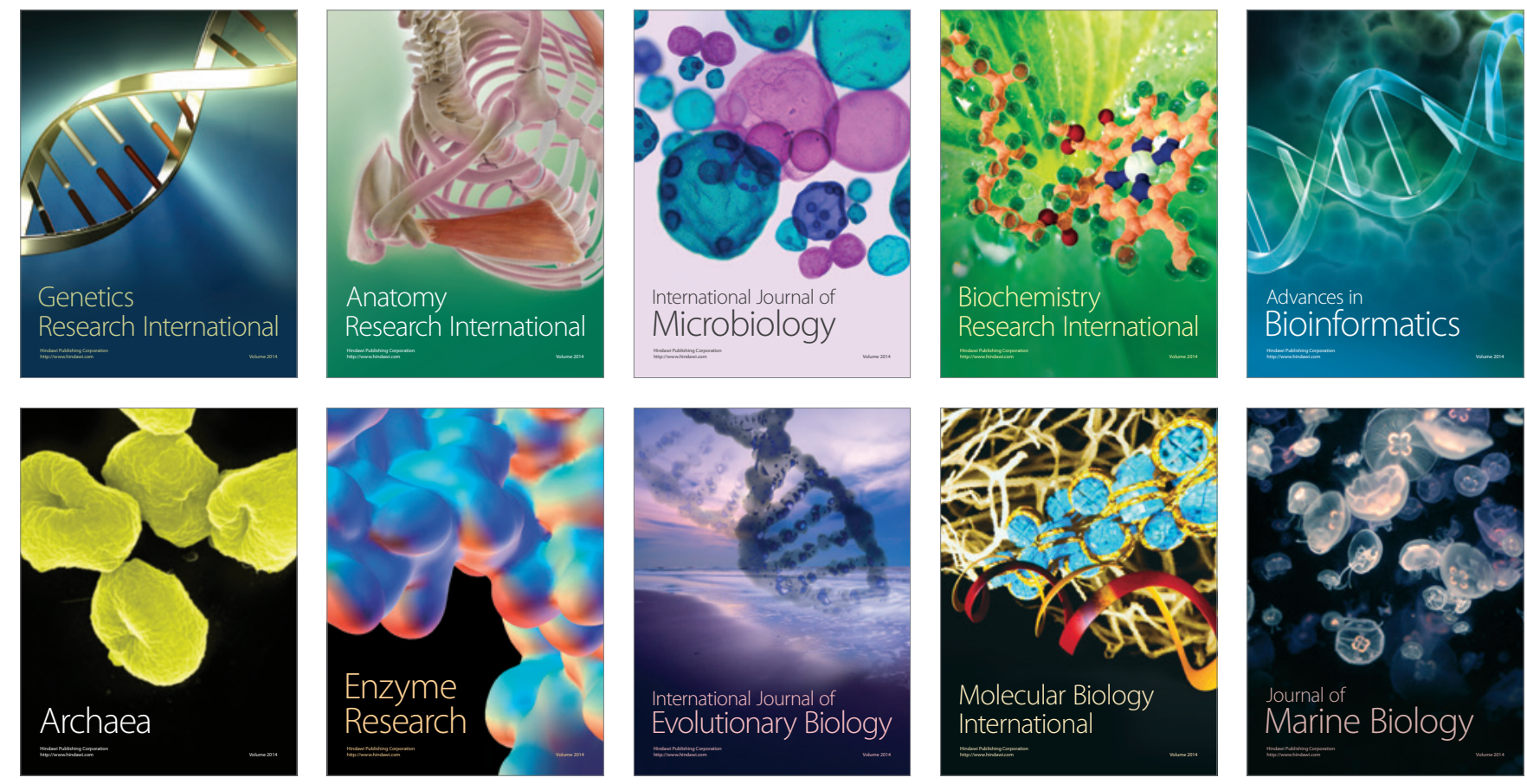